\title{
Burnout syndrome among psychiatrists in Egyptian mental health hospital
}

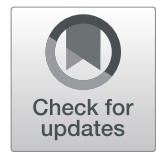

\author{
Mohamed Abu zied ${ }^{1}$, Mohamed Fekry ${ }^{1}$, Nesreen Mohsen ${ }^{1}$, Mahmoud Morsy ${ }^{1,2^{*}}$, Doha El Serafy ${ }^{1}$ and \\ Mohamed Salah ${ }^{3}$
}

\begin{abstract}
Background: Burnout is a special type of work-related stress-a state of physical or emotional exhaustion that also involves a sense of reduced accomplishment and loss of personal identity (Lancet 388:2272-2281, 2016). Burnout is a syndrome of state of emotional exhaustion, depersonalization, and feelings of low personal accomplishment (Maslach C and Jackson S, Maslach burnout inventory manual, 1986). Burnout syndrome leads to severe problems. It may cause psychosomatic problems, family and marital conflicts, and substance misuse and also may result in complications such as late coming and early leaving of employees, quitting, having frequent medical reports, and the lack of productive and constructive ideas and criticism in the occupational settings (Sayil et al, Kriz Dergisi, Cilt 5, Say 1:2 ss.71-77, 1997).

So, we aimed to estimate the rate of occurrence of burnout among a sample of psychiatrists in one of the governmental psychiatric hospitals in Egypt.

Results: Result shows that $56.2 \%$ of study sample were males and $43.8 \%$ were females. Their mean age was $32 \pm 6$, and $57.5 \%$ were married and $42.5 \%$ were single. Mean score of emotional exhaustion was $28.25 \pm 10.45$ (high).

Mean score of depersonalization was $8.5 \pm 6.1$ (moderate). Mean score of personal accomplishment was $31.78 \pm 8.5$ (low)

Conclusion: Burnout rate among psychiatrists of Al-Abbassia Hospital is higher than in western countries. Marriage and sleeping in home have protective effects. There is a significant association between high number of working hours per week and quality of the relationship with seniors and burnout.
\end{abstract}

Keywords: Burnout, Emotional exhaustion (EE), Depersonalization (DP), Personal accomplishment (PA)

\section{Background}

Job burnout is a special type of work-related stress-a state of physical or emotional exhaustion that also involves a sense of reduced accomplishment and loss of personal identity. "Burnout" is not a medical diagnosis. Some experts think that other conditions, such as depression, are behind burnout. Some research suggests that many

\footnotetext{
* Correspondence: mahmoudmorssy84@hotmail.com

'Department of Neuropsychiatry, Institute of Psychiatry, The WHO

Collaborating Center for Research \& Training in Psychiatry, Ain Shams

University, Abbasseyia, Ramses Street Extension, Dair Al-Malak, P.O. Box:

11657, Cairo, Egypt

${ }^{2}$ Faculty of Medicine, Ain Shams University, Abbasseyia, Cairo, Egypt

Full list of author information is available at the end of the article
}

people who experience symptoms of job burnout do not believe their jobs are the main cause [52].

Lee and Ashforth [22] defined burnout as a syndrome of state of emotional exhaustion, depersonalization, and feelings of low personal accomplishment. This definition was based on Maslach and Jackson's [27] work.

Emotional exhaustion is used to describe state of feeling emotionally overextended and exhausted by their work. It may be presented by tiredness, somatic symptoms, decreased emotional resources, and a feeling that one has nothing left to give to others. Depersonalization defines people who develop negative, cynical attitudes and impersonal feelings towards their clients, treating them as objects. Reduced personal accomplishments 
denote feelings of incompetence, inefficiency, and inadequacy. The higher the emotional exhaustion and depersonalization scores, and the lower the personal accomplishment score, the more a physician could be suffering from burnout [37].

Burnout syndrome leads to severe problems. Personally, it may give rise to psychosomatic problems, family and marital conflicts, insomnia, fatigue, anger and irritability, and alcohol or substance misuse. As for organizational contexts, burnout may result in complications such as late coming and early leaving of employees, quitting, having frequent medical reports, and the lack of productive and constructive ideas and criticism in the occupational settings [47].

The nature of the medical profession makes the physicians prone to burnout; taking care of the patients, being responsible of making the right diagnosis and planning for the proper management, overnight shifts, working for long hours, continuous medical education, and work/home conflicts all are stressors present in physicians' lives and expose them to burnout [35].

Psychiatrists have more stressful life among physicians, as they use themselves as "tools" in their profession and experience a range of powerful emotions in their clinical work. The doctor-patient relationship in itself evokes emotions such as the need to rescue the patient, a sense of failure and frustration when the patient's illness progresses or does not respond to treatment, feelings of powerlessness against illness and its associated losses, grief, fear of becoming ill oneself, or a desire to separate from and avoid patients to escape these feelings. In addition, the personal nature of the relationship psychiatrists have to develop with their patients, and these emotions are likely to be intensified in their context $[20,30]$.

Psychiatrists are also exposed to external stressors due to work environment. Rapidly changing ways of service delivery, the gap between the academic training and the clinical practice, and the increasingly complex administrative and legal frameworks are of major influence on psychiatrists [20].

Many studies were concerned by the susceptibility of psychiatrists to burnout. Psychiatrists used to face crowded inpatient wards, work overload, responsibility for patients and relapsing patients, identified violence and the fear of violence, high work demands without adequate resources, poorly defined roles of consultants, responsibility without authority, overt bureaucracy and inability to change the system, conflict between responsibility towards employers versus towards the patients, isolation of consultants in community of mental health teams, lack of free time, and an increasing community criticism to the mental health services [10, 41, 48].

These factors may be either responsible for premature retirement by specialist psychiatrists or be reasons for juniors to avoid psychiatry as a career option [29].
As regards the relationship between job satisfaction and burnout, most studies showed an inverse relationship between the two variables in the majority of professions [7, 36].

On the contrary, psychiatrists can continue to enjoy their work and consistently score high in job satisfaction surveys $[40,43]$. These findings indicate that psychiatrists are devoted and passionate towards their work regardless its association with burnout [20].

This study is aiming to estimate the rate of burnout syndrome among psychiatrists of $\mathrm{Al}$ Abbasia Mental Health Hospital in Egypt and factors related to it.

\section{Methods}

\section{Participants}

The study was conducted at Al-Abbasia Mental Health Hospital, Cairo, Egypt. El Abbasia Hospital is the oldest and largest psychiatric hospital in Egypt, and it is operated by the Health Ministry's General Secretariat of Mental Health and Addiction Treatment. It currently accommodates 1504 beds distributed among 46 departments; this is besides the different outpatient clinics and the daycare treatment and rehabilitation center.

The total number of physicians at Al-Abbassia Mental Health Hospital is 128 physicians, 108 of them are psychiatrists. Subjects who were excluded from the study were distributed as follows: 16 who have not completed their internship year, have not spent at least 6 months working in the specialty of clinical psychiatry, or have no regular attendance to the hospital; 15 psychiatrists refused to fill the questionnaires; and 2 did not complete the whole data. The final sample included 73 psychiatrists; those were selected over a period of 1 month. A written consent was taken by the subjects after discussing the aim of the study with them. All subjects were subjected to a preliminary short interview aiming to build rapport, ensuring consent and confidentiality of gathered data.

Ethical approval for the study was granted by the Ethical and Research Committee of Faculty of Medicine, Ain Shams University, as well as the Ethical Committee of the General Secretariat of Mental Health and Addiction Treatment, Ministry of Health and Population, Egypt.

\section{Procedure and instruments}

All the psychiatrists were examined using a semistructured sheet prepared by the researchers, including socio-demographic data like gender, age, marital status, smoking, and other data like years of experience, work nature, the time spent to reach the hospital, working hours per week, working days per week, overnight shifts per month, number of patients interviewed per day, presence of private work, chronic illness or special family circumstances, presence of friendships in the hospital 
outside the frame of the work, receiving phone calls from the patients, satisfaction of the family with the specialty, and relations with seniors. The Maslach Burnout Inventory (MBI) [27] is a 22-item questionnaire that is considered to be the gold standard measure for burnout. It evaluates the three independent dimensions of burnout: emotional exhaustion (range $0-54$ ), depersonalization (range 0-30), and personal accomplishment (range 0-48). Table 1 shows the cutoff scores of total scores and subscales and mid, moderate, and high score cutoffs $[25,28]$.

An Arabic version of The Maslach Burnout Inventory (MBI) was purchased online with a license to use and reproduce, and it was printed with the semi-structured sheet to be distributed in Al-Abbassia Hospital. This Arabic version was translated by Rouba Bakkour on May 20, 2003. It was translated and reproduced by special permission of the Publisher, Maslach Burnout Inventory-HSS by Christina Maslach and Susan E. Jackson, Copyright 1986 by CPP, Inc.

\section{Statistical analysis}

Data was coded, entered, and analyzed using SPSS for Windows version 20. Continuous variables such as age were expressed as mean and standard deviation, whereas categorical variables such as gender were presented as frequencies. Analysis of variance (ANOVA) methods were used for categorized independent variables. Chisquare is used to estimate the difference in unpaired categorical variables and Student's test to estimate the difference in numerical variables. A $P$ value $<.05$ was considered as statistically significant. Linear regression was used to calculate the standardized coefficients of regression and detect the significant variables and their effect on outcome (MBI subscales). Pearson's correlation was used to measure the linear correlation between two variables.

\section{Results}

This study investigates the rate of the burnout syndrome at the level of its three dimensions among psychiatrists of Al-Abbassia Mental Health Hospital. Also, the relation between the sociodemographic data as well as work environment and the burnout syndrome had been studied. A total of 73 psychiatrists were recruited from the concerned hospital to participate in the study.

Table 1 Classification of Maslach Burnout Inventory

\begin{tabular}{llll}
\hline Burnout level & $\begin{array}{l}\text { Emotional } \\
\text { exhaustion }\end{array}$ & Depersonalisation & $\begin{array}{l}\text { Personal } \\
\text { accomplishment }\end{array}$ \\
\hline High & $\geq 27$ & $\geq 10$ & $0-33$ \\
Moderate & $19-26$ & $6-9$ & $34-39$ \\
Low & $0-18$ & $0-5$ & $\geq 40$ \\
\hline
\end{tabular}

Socio-demographic data shows that $56.2 \%(N=41)$ of the study sample were males and $43.8 \%(N=32)$ were females. Their mean age was $32 \pm 6 ; 57.5 \%(N=42)$ were married, and $42.5 \%(N=31)$ were single.

Most of study participants were non-smokers (93.2\%), and just $6.8 \%(N=5)$ were smokers. $20.5 \%(N=15)$ had chronic illness [4.1\% $(N=3)$ had hypertension, and $4.1 \%$ $(N=3)$ had depression, while autoimmune disorders, e.g., systemic lupus and rheumatoid arthritis, were present in $2.7 \%(N=2)]$.

Mean number of nights spent with the family was $5 \pm 2$ nights, and $2.7 \%$ ( 2 female psychiatrists) were pregnant.

12.3\% $(N=9)$ had the burden of infant care, and the rest had not. $4.1 \%(N=3)$ has other special circumstances (not mentioned).

When the study subjects were divided into different subgroups as regard (sex, marital status, smoking, special needs, and presence of chronic illness), there was no significant difference regarding the first two variables (sex and marital status) and $P$ value $>0.05$ in both, while there was significant differences between study subgroups regarding smoking variable (the majority was non-smokers), regarding special circumstances (the majority did not report special circumstances), and regarding the variable of chronic illness (the majority had no chronic illness); in those variables, $P$ value was $<0.05$.

As regards work environment data mean, minutes consumed by the psychiatrists to go to the hospital from home was $69 \pm 47 \mathrm{~min}$. Mean number of years spent in the specialty of psychiatry was $6 \pm 5$ years. Mean number of patients examined by the psychiatrist daily was 14 \pm 9 patients. Mean number of working days per week for the psychiatrist was $5 \pm 1$ days per week. Mean number of working hours per week for the psychiatrist was $45 \pm 23 \mathrm{~h}$ per week. Mean number of overnight shifts taken by the psychiatrist per month was $4 \pm 5$ shifts per month (Table 2).

$56.2 \%(N=41)$ of the study sample are working in the field of general psychiatry, and $43.8 \%(N=32)$ are not working in the field of general psychiatry.

$54.8 \%(N=40)$ of the psychiatrists work in psychiatric subspecialties (addiction, child psychiatry, adolescents psychiatry, geriatric psychiatry, community psychiatry,

Table 2 Work environment data

\begin{tabular}{ll}
\hline Work related data & Mean \\
\hline Time to work in minutes & $69 \pm 47$ \\
Number of working years & $6 \pm 5$ \\
Average number of patients/day & $14 \pm 9$ \\
Number of working days & $5 \pm 1$ \\
Number of working hours & $45 \pm 23$ \\
Average number of shifts & $4 \pm 5$ \\
\hline
\end{tabular}


forensic psychiatry) with the percentages shown in Fig. 1, and $45.2 \%(N=33)$ do not work in psychiatric subspecialty.

Also, concerning the nature of the work of the psychiatrists in Al-Abbassia Mental Health Hospital, it was found that $84.9 \%(N=62)$ of the psychiatrists involved in the study work in the outpatient clinic and $15.1 \%(N=11)$ do not work in the outpatient clinic, $78.1 \%(N=57)$ work in inpatient wards and $21.9 \%(N=16)$ do not work in inpatient wards, and $4.1 \%(N=3)$ perform field visits outside the hospital and $95.9 \%(N=70)$ do not perform field visits outside the hospital.

$8.2 \%(N=6)$ work in the psychiatric emergency room, and $91.8 \%(N=67)$ do not work in the psychiatric emergency room.

As regards the burnout rating of the psychiatrists (Table 3), mean score of emotional exhaustion for the psychiatrists was $28.25 \pm 10.45$ (high). Mean score of depersonalization for the psychiatrists was $8.5 \pm 6.1$ (moderate). Mean score of personal accomplishment for psychiatrists was $31.78 \pm 8.5$ (low) (Fig. 2).

On the subscale of emotional exhaustion, $58.9 \%$ of the study sample had high scores of emotional exhaustion, $27.4 \%$ of the study sample had moderate scores of emotional exhaustion, and $13.7 \%$ of the study sample had low scores of emotional exhaustion.

On the subscale of depersonalization, $42.5 \%$ of the study sample had low scores of depersonalization, $38.4 \%$ of the study sample had moderate scores of depersonalization, and $19.1 \%$ of the study sample had high scores of depersonalization.

On the subscale of personal accomplishment, $45.2 \%$ of the study sample had high scores of personal accomplishment, $27.4 \%$ of the study sample had moderate scores of personal accomplishment, and $27.4 \%$ of the study sample had low scores of personal accomplishment,

As regards the relation between burnout syndrome and socio-demographic variables, comparison of MBI dimension scores according to gender showed that mean score of emotional exhaustion among female psychiatrists was
Table 3 Mean scores of MBI subscales

Maslach Burnout Inventory (MBI)

\begin{tabular}{llll}
\hline Subscale & $\begin{array}{l}\text { Emotional } \\
\text { exhaustion }\end{array}$ & $\begin{array}{l}\text { De- } \\
\text { personalization }\end{array}$ & $\begin{array}{l}\text { Personal } \\
\text { accomplishment }\end{array}$ \\
\hline Mean & $28.25 \pm 10.45$ & $8.55 \pm 6.16$ & $31.78 \pm 8.54$ \\
\hline
\end{tabular}

higher than the score among the male group $(29.2 \pm 11.3$, $27.5 \pm 9.8)$ respectively, but the difference between both groups was not statistically significant $(P$ value $=0.473)$.

Mean score of depersonalization among male psychiatrists was higher than the score among the female group, but the difference between them was not statistically significant $(P$ value $=0.314)$.

Mean scores of personal accomplishment among males and females was relatively the same.

Comparison of MBI dimension scores according to marital status showed that mean score of emotional exhaustion among married psychiatrists was higher than the score among the single group $(29.2 \pm 9.2,26.9 \pm$ 11.9) respectively, but the difference between both groups was not statistically significant $(P$ value $=0.361)$.

Mean score of depersonalization among married psychiatrists was lower than the score among the single group $(7.9 \pm 5.9,9.4 \pm 6.4)$ respectively, but the difference between both groups was not statistically significant $(P$ value $=0.303)$.

It was found that being married is statistically significantly associated with higher scores of personal accomplishment; mean score of personal accomplishment among married psychiatrists $(33.7 \pm 8.3)$ was higher than the mean score among the single group $(29.1 \pm 8.2)$ with $P$ value $=0.022$.

Comparison of MBI dimension scores according to age showed there were no significant differences in mean scores of the three dimensions of burnout between the different age groups.

Regarding the effect of special family condition (pregnancy, infant care, disability, a person in family needing

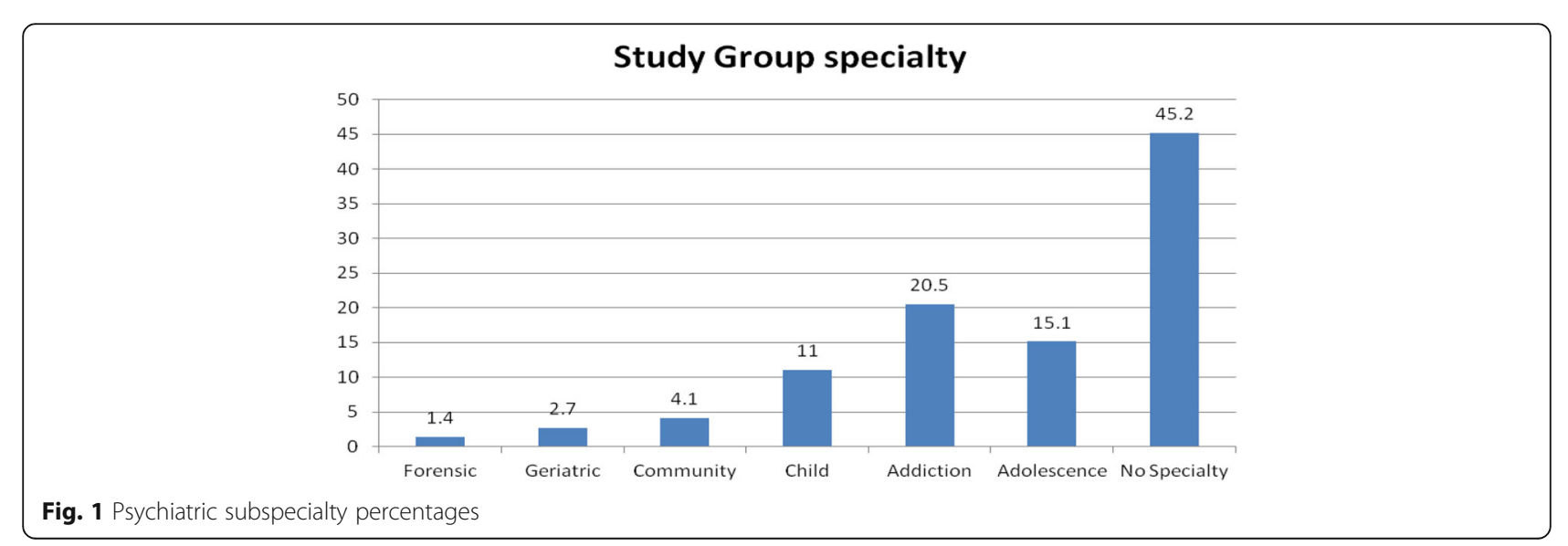




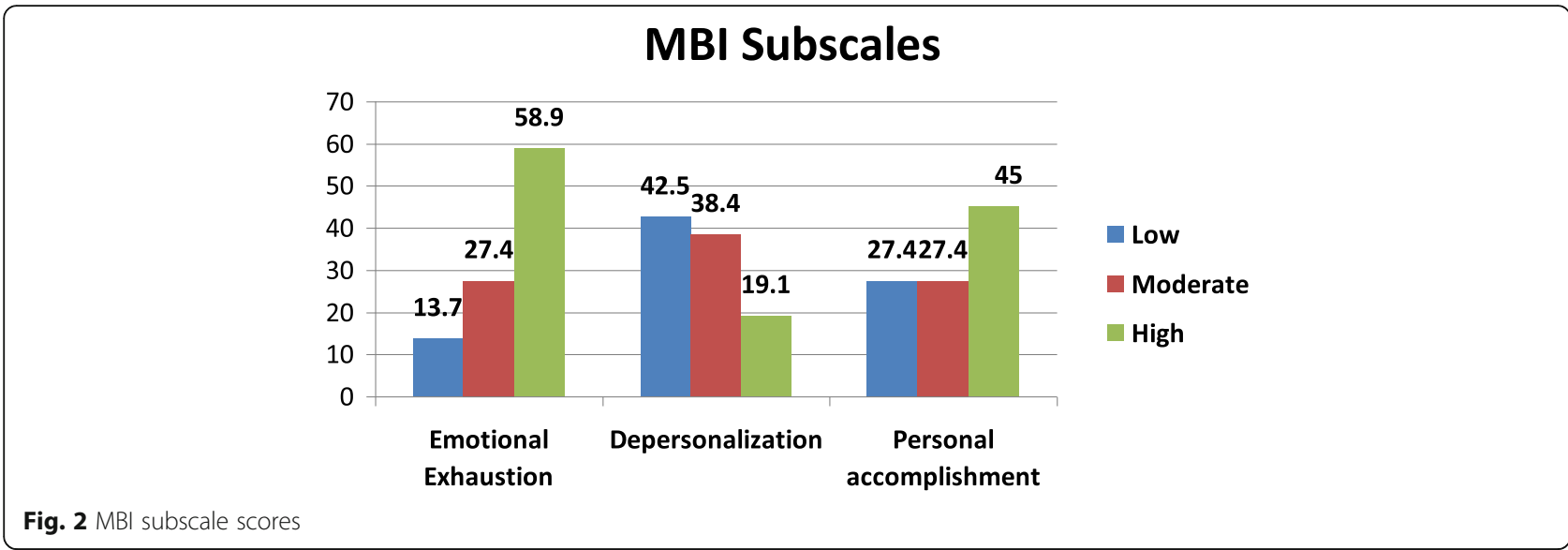

special care and others) on MBI subscales, there were no significant differences in the mean scores of the three dimensions of burnout.

Regarding the effect of chronic illness on MBI subscale, there were no significant differences in the mean scores of the three dimensions of burnout between the group of psychiatrists who has chronic illness and the group who has not.

As regards the effect of work satisfaction on MBI subscales, according to the study, $5.4 \%(N=4)$ of the sample are not satisfied with being psychiatrists and $94.6 \%$ $(N=69)$ are satisfied. There were no significant differences in the mean scores of the three subscales of burnout between the group of psychiatrists satisfied with being psychiatrists and the group who is not satisfied.

According to the study, $32.8 \%(N=24)$ of the psychiatrists involved in the study reported that their familiesin general-are not satisfied with choosing psychiatry as a specialty, and $67.2 \%(N=49)$ of the psychiatrists reported that their families-in general-are satisfied with choosing psychiatry as a specialty.

Having a family not satisfied by the one's specialty is significantly associated with higher scores of depersonalization and lower scores of personal accomplishments.

\section{C-relation between burnout syndrome and work environment variables}

Comparison of $\mathrm{MBI}$ dimension scores according to working years

Number of working years (years of experience in psychiatry) was divided into three groups: $\leq 10$ years, $1-20$ years, and above 20 years. There were no significant differences in the mean scores of the three dimensions of burnout between the three groups.

The mean score of emotional exhaustion and depersonalization among psychiatrists who work with administrative and paper works beside their clinical work was higher but was not statistically significant.
Mean score of personal accomplishment among psychiatrists who work with administrative and paper works beside their clinical work was significantly higher than the score of psychiatrists who just work with clinical work with statistically significant difference.

No psychiatric subspecialty was found to have a statistically significant association with high level of burnout.

There were no statistically significant associations between average daily numbers of patients or working days per week or average shifts per month and the scores of the subscales of burnout (Table 4).

It was found that the number of working hours per week is statistically significantly positive correlation associated with the level of depersonalization. It was found also that the number of nights spent with the family per week is statistically significantly positive correlation associated with the level of personal accomplishment.

There are no significant differences in the mean scores of the three subscales of burnout between the group of psychiatrists who work at private work and the group who does not.

According to the study, $95.9 \%(N=70)$ of the study sample have chosen the specialty of psychiatry by their free will and $4.1 \%(N=3)$ was forced to take this specialty due to the rules of medical residency and other causes; there are no significant differences in mean scores of the three subscales of Burnout between both groups.

To study the effect of the presence of work friends on MBI subscales, according to the study, $83.5 \%(N=61)$ of the study sample have friends in the hospital outside the work frame and $16.5 \%(N=12)$ do not have. Presence of friendships outside the work frame in the hospital does not have a statistically significant effect on burnout subscale scores.

According to the study, $63 \%(N=46)$ of the study sample receive calls from patients on their private phone and $37 \%(N=27)$ do not receive calls from patients on their private phone; there are no significant differences 
Table 4 Correlation between subscales and work variables

\begin{tabular}{lllllll}
\hline & & Average number of patients & Working days & Working hours & Average shift & Number of family nights \\
\hline Emotional exhaustion & $r$ & -0.002 & 0.121 & 0.220 & 0.079 & 0.147 \\
& $P$ value & 0.985 & 0.308 & 0.061 & 0.505 & 0.216 \\
Depersonalization & $r$ & 0.085 & 0.142 & $0.248^{*}$ & 0.192 & -0.054 \\
& $P$ value & 0.475 & 0.232 & 0.034 & 0.103 & 0.653 \\
Personal accomplishment & $r$ & 0.073 & 0.184 & 0.017 & -0.207 & $0.334^{* *}$ \\
& $P$ value & 0.541 & 0.120 & 0.885 & 0.079 & 0.004 \\
\hline
\end{tabular}

in the mean scores of the three subscales of burnout between both groups.

It was found that the quality of relations with seniors in the hospital has a statistically significant effect over all three subscales of burnout.

Good relations with seniors was significantly associated with lower levels of emotional exhaustion and vice versa, lower levels of depersonalization and vice versa, and higher levels of personal accomplishment and vice versa.

Multiple regression analysis was conducted to examine the relationship between emotional exhaustion among the studied psychiatrists and various potential predictors. The multiple regression model shows that the variable "psychiatrist's relation with seniors" had significant negative regression, indicating psychiatrists with good relations with seniors were expected to have lower emotional exhaustion score on the MBI subscale, after controlling for the other variables in the model. Other variables did not contribute to the multiple regression models. This result indicates the statistical robustness of "the relation with seniors" variable.

Multiple regression analysis was conducted to examine the relationship between depersonalization among the studied psychiatrists and various potential predictors. It showed that the variables "psychiatrist's relation with seniors" and "number of working hours" had significant negative regression, indicating psychiatrists with good relations with seniors and psychiatrists with low number of working hours were expected to have lower depersonalization score on the MBI subscale, after controlling for the other variables in the model. Other variables did not contribute to the multiple regression model.

Multiple regression analysis was conducted to examine the relationship between personal accomplishment among the studied psychiatrists and various potential predictors. It showed that there is no significant variable, which means that no variable was strong enough to present itself in personal accomplishment model.

\section{Discussion}

Physician burnout is an important topic for investigation, because it may lead to negative consequences for both physicians and patients. High levels of burnout are associated with decreased emotional well-being, increased absenteeism, and a reduction in personal accomplishments and ineffectiveness, as well as an increase in risk factors for cardiovascular diseases and risk for additional diseases [12, 31, 51].

The aim of the current study was to estimate the rate of occurrence burnout syndrome among psychiatrists of Al-Abbassia Mental Health Hospital, also to study the socio-demographic characteristics of psychiatrists suffering from burnout syndrome and to assess associated factors that increase the burnout syndrome and propose different recommendations to protect from it.

The total number of physicians at Al-Abbassia Mental Health Hospital is 128 physicians, 108 of them were psychiatrists; all the psychiatrists were offered to fulfill the questionnaires including the Arabic version of Maslach Burnout inventory and the semi-structured questionnaire to complete their socio-demographic and workrelated data, 18 psychiatrists did not meet the inclusion criteria, 15 psychiatrists refused to fill the questionnaires, and 2 did not complete the whole data. The rest 73 psychiatrists were included in the study.

Regarding scores of MBI (prevalence of burnout syndrome), the mean score of emotional exhaustion for the psychiatrists in our study was higher than that of a similar Italian study that measured burnout among psychiatrists of Milan city in 2009. Mean score of depersonalization for the psychiatrists was higher than that of the same Italian study. Mean score of personal accomplishment for psychiatrists was lower than that of the Italian study [6].

Our scores also indicated severe level of burnout in higher percentages than another similar British study [39].

The high scores of burnout subscales in our study compared to the Italian and British studies may be due to the different work circumstances between psychiatrists in the different countries and being more distressing here in Egypt; higher patient flow rates in clinics and higher percentages of ignorance and illiteracy among patients may be stressors that explains higher burnout in the current study.

Regarding the relation between burnout syndrome and socio-demographic variables, there was no significant association between gender difference and the severity of burnout syndrome in our study, that is consistent with 
previous Egyptian study that measured burnout among residents of Ain Shams University from all the medical specialties [37]. But contradictory with study in New Zealand that targeted all the registered psychiatrists in the country, in this study, personal accomplishment was significantly higher in males than in females [21] which means that females are more prone to burnout than males. This contradiction can reveal higher resilience for the Egyptian female in front of work stressors or may be due to harder job circumstances for female psychiatrists in New Zealand.

Being married was significantly associated in our study with higher level of personal accomplishment thus lower level of burnout syndrome; that could be due to the support given by the spouse or the idea that one has achieved a social and a life milestone by getting married and establishing a family and maybe also due to social stress that could be put on single subjects in our middle eastern society.

In our study, marital status was not related to emotional exhaustion (EE) and depersonalization (DP). Our results are comparable to those of New Zealand national study in which being single was associated with high levels of EE but not related to levels of DP and personal accomplishment (PA). However, it still agrees with our study that marriage is protective against burnout [21]. Contradictory to these results were the results of Osama [37], and this can be explained by the underrepresentation of the married subjects reported in Osama [37].

There was no significant association between age and the severity of burnout syndrome in our study; this is consistent with the results of Osama [37] in Egypt and contradictory to results of Boyas et al. [5] and Kumar [21] in the USA and New Zealand, in which respondents who were younger than 40 years were found to report significantly higher scores on EE than other age groups, explaining that with lack of experience and coping techniques and being more stressed and idealistic.

Differences in results may be explained by differences in challenges faced by young psychiatrists between different cultures and countries and may be due to the presence of supervisory support from seniors in places more than others or may be due to less professional responsibilities on juniors here in Egypt.

There was no significant association between having special family conditions or burdens (pregnancy, infant care, disability, a person in family needing special care, and others) and the severity of burnout syndrome in our study; this is inconsistent with the results of Almberg et al. [2] who found significant association between burnout and caring of demented elderly people in Sweden, and inconsistent with Lindström et al. [26] who found significant association between burnout and parenting chronically ill children in Sweden [4] who reviewed in Journal of Advanced Nursing the significant association between burnout and parenting intellectually disabled child.

Differences in results can be clearly explained with underrepresentation of psychiatrists with family burdens in the current sample.

There was no significant association between having chronic illness and the severity of burnout syndrome in our study, which is inconsistent with the results of a huge study in the general population in Finland who found that physical illnesses are associated with increase of all three dimensions of burnout and that burnout is associated with musculoskeletal diseases among women and with cardiovascular diseases among men [16].

Our study results are also inconsistent with the results of Melamed et al. [31] who reviewed the relation between burnout and chronic illness especially cardiovascular disease and cardiovascular-related events and found strong association between them. This contradiction is mostly due to underrepresentation of psychiatrists with chronic illness in the sample or due to different age group.

Our results showed no relation between job satisfaction and burnout in contradiction to most of the literatures like Kumar [21], Slayers et al. (2013), and Renzi et al. [42] who emphasized the inverse relation between burnout and job satisfaction; this contradiction is clearly due to underrepresentation of psychiatrists not satisfied with their job in the sample and because we did not use assessment tools for the assessment of job satisfaction.

We found significant association between family satisfaction and the severity of burnout specifically depersonalization and personal accomplishment. Poor family satisfaction was associated with high scores of depersonalizations and low scores of personal accomplishments, thus higher burnout.

Regarding relation between burnout syndrome and work environment variables, there was no significant association between number of working years or years of experience and the severity of burnout syndrome in our study. This is consistent with another similar studies like Osama [37] and Lešić et al. [24] but inconsistent with another similar studies like Montero-Marin et al. (2011), Ross et al. [45], Whittington [53], and Kumar [21].

Some of these studies found that length of the service in the organization showed a direct linear association with the burnout, to the extent that the longer the service, the greater the likelihood of having high burnout profile (Montero-Marin et al. 2011 [45];).

But this variable is ambivalent in its relationship with burnout syndrome in general; their association could be positive in both directions as some studies proposed an inverse association $[21,53]$.

This contradiction may be due to the differential impacts of the various types of organizations on their 
employees, some of which offer protection from the development of the syndrome, while other induce it, or may be the point is related to the culture of the place of the study about seniority and different concepts of seniority and the higher responsibilities of the more experienced staff.

In our study, we found that the scores of EE and DP of psychiatrists performing administrative paperwork were higher than the rest of psychiatrists but without statistically significant difference; this is consistent with the results of a study which revealed levels of EE and DP were positively related to doing administrative paperwork but with $P$ value $>0.05$ [46], and also consistent with Gundersen [14] in which the relation was statistically significant with $P$ value $<0.05$.

On the dimension of personal accomplishment, we found that there is significant association between administrative paperwork and high PA scores which is protective against burnout; this is inconsistent with Rupert and Morgan [46] and Bruce et al. [8] in which PA was negatively related to performing administrative paperwork besides the original clinical work. This contradiction in the results of PA is because in some places like here in Egypt, having administrative role gives more prestige and influence and make you effective on a wider base.

There was no significant association between the subspecialty of the psychiatrist (child, adolescents, addiction, geriatrics, community, forensic) and the severity of burnout syndrome in our study; this is consistent with the results of Korkeila et al. [18] who compared burnout scores of child psychiatrists to other psychiatrists, and consistent also with Volker et al. [50] and Vilardaga et al. [49] who compared burnout scores of a sample of addiction treatment service staff to other healthcare service staffs.

Our study results are inconsistent with surprising results of a similar study in Australia [15] who found that forensic psychiatry staff displayed lower burnout and higher job satisfaction than their counterparts from the mainstreamed psychiatric services.

It is inconsistent also with Benbow and Jolley [3] who found that old age psychiatrists (geriatric psychiatrists) have higher scores of burnouts than other mainstream psychiatrists. The contradiction with the studies of forensic and old age psychiatrists is mostly due to the underrepresentation of these subspecialties in our study.

The average number of patients interviewed by the psychiatrist in our study was $14 \pm 9$ patients per day. There was no significant association between the number of patients and the severity of burnout syndrome in our study; this is consistent with the results of Kumar [21] and inconsistent with the results of Pérez et al. [38] and Grassi and Magnani [13] who found positive relation between number of patients interviewed and severity of burnout.
Some studies found opposite results in the form of negative relation between the number of patients and severity of burnout as the percent of burnout increased with the decrease in number of consulted patients $[19,23,33]$.

This contradiction between studies is mostly due to the issue of actual workload versus perceived workload as burnout development perceived workload is the main determinant, while the actual case load and work times contribute indirectly to burnout, through perceived workload, in another word: burnout is not about workload, it is about the perception of workload; if it is a positive perception, it will not elicit burnout, and if it is a negative perception, it will elicit burnout [19].

We found a significant association between working hours of the psychiatrist per week and burnout specifically the subscale of depersonalization; this is consistent with all the similar studies that could be gathered by the researcher like Osama [37], Montero-Marín et al. [32], Al-Dubai and Rampal [1], Lee and Ashforth [23], Caruso [9], and Rogers et al. [44].

The number of hours worked per week was associated directly and linearly with the burnout in such a way that when the number of hours was increased, so was the risk of developing this burnout profile.

The core results of the previous similar studies suggest that this variable seems to be the key factor in the configuration of this profile and could contribute to the development of the syndrome by increasing worker exhaustion levels.

In our study, we found a significant positive association between numbers of night spent with the family by the psychiatrist and scores of personal accomplishment, thus a negative relation between this variable and burnout; it means that sleeping in home with your family has protective effect against burnout statistically; this is consistent with the results of Jensen and Rundmo [17] and Dyrbye et al. [11] which revealed positive associations between the number of overnight shifts or overnight staying out on a side, and work family conflict and burnout on another side.

This can show the emotional and psychological positive effect of spending night at home and sleeping in the own bed on the relief of job stress.

The prominent finding of our study was about the effect of quality of relation with seniors/supervisors on burnout syndrome as we found significant effect for it on the whole three subscales of burnout; better relationships with seniors was significantly associated with lower levels of EE and DP and higher levels of PA. This is consistent with similar studies which showed that work climate satisfaction was in negative correlation with all three dimensions of burnout syndrome, which shows that workers who are less satisfied with the work climate display a higher degree of burnout [34]. 
These results seem to indicate a need for managers to concentrate their efforts on increasing supervisory skills as a means of preventing burnout among their employees/psychiatrists. Such strategies should include training supervisors in recognizing signs of burnout and providing ways and means of assisting employees in coping with and/or preventing burnout symptoms.

Multiple regression analysis was done on the sociodemographic and work-related variables to examine the robustness of these variables. It was found that quality of relationship with seniors has the controlling effect on emotional exhaustion subscale. Quality of relationship with seniors and number of working hours have the controlling effect on depersonalization subscale, yet no variable was strong enough to present itself in the model of personal accomplishment.

Another study that shares our study in the robustness of relationship with seniors in regression model is the Italian study performed on the psychiatrists of Milan city (Bressi 2009) which showed its significance specifically on personal accomplishment subscale; the Italian study showed also significance of job satisfaction in the regression model of whole three burnout subscales, which did not appear in our regression model that could be due to the underrepresentation of the not satisfied psychiatrists in our sample and also because we did not use assessment tools to assess job satisfaction as it was not our focus of interest in the methodology of the current study.

\section{Limitations}

1. We had a limited sample size, so there was underrepresentation for some categories in the sample like psychiatrists having family burden, psychiatrists having chronic illness, psychiatrists not satisfied with the specialty, forensic psychiatrists, and geriatric psychiatrists, so these variables were not correlated to burnout properly.

2. Some psychiatrists refused to participate in the study; we do not have sufficient data or information about those psychiatrists whose rejection could be due to causes related to being actually suffering from burnout syndrome.

3. This is a cross-sectional study, so longitudinal studies and follow-up data may be needed in the future.

\section{Conclusion}

Burnout is a highly important issue to be taken care of in any profession dealing with people including medical professions and psychiatry in particular and have its effects on nations' productivity and service quality. $\checkmark$ Burnout rate among psychiatrists of Al-Abbassia Hospital is high, and higher than its rate among psychiatrists in western countries.

$\checkmark$ Marriage and sleeping in home with the family have protective effects against burnout statistically.

$\checkmark$ There is a significant association between high number of working hours of the psychiatrist per week and burnout.

$\checkmark$ Perceived stress and workload have more significant role in suffering from burnout than the actual workload and patients' rate.

$\checkmark$ Quality of the relationship with seniors has fundamental effect on burnout among psychiatrists, bad relationship with seniors is a predictor of high level of burnout, and good relationship with seniors is protective against burnout.

\section{Abbreviations}

MBI: Maslach Burnout Inventory; EE: Emotional exhaustion;

DP: Depersonalization; PA: Personal accomplishment

\section{Acknowledgements}

Not applicable

Reference number of ethical committee approval is notavailable. A written consent was taken by the subjects after discussing the aim of the study with them.

\section{Authors' contributions}

All authors contribute in writing the manuscript. MA and MF made a design of the work. NM and MS did the analysis and interpretation of data. MM and DE had drafted the work and substantively revised it. The authors have read and approved the final manuscript.

Funding

Not applicable

Availability of data and materials

Available when needed

Ethics approval and consent to participate

Ethical approval for the study was granted by the Ethical and Research Committee of Faculty of Medicine, Ain Shams University, as well as the Ethical Committee of the General Secretariat of Mental Health and Addiction Treatment, Ministry of Health and Population, Egypt.

Consent for publication

Not applicable

Competing interests

No conflict of interest.

Author details

'Department of Neuropsychiatry, Institute of Psychiatry, The WHO Collaborating Center for Research \& Training in Psychiatry, Ain Shams University, Abbasseyia, Ramses Street Extension, Dair Al-Malak, P.O. Box: 11657, Cairo, Egypt. Faculty of Medicine, Ain Shams University, Abbasseyia, Cairo, Egypt. ${ }^{3}$ ElAbassia Mental Hospital, Cairo, Egypt.

Received: 8 January 2020 Accepted: 27 March 2020

Published online: 10 June 2020

References

1. Al-Dubai SAR, Rampal KG (2010) Prevalence and associated factors of burnout among doctors in Yemen. J Occup Health 52(1):58-65 
2. Almberg B, Grafström M, Winblad B (1997) Caring for a demented elderly person - burden and burnout among caregiving relatives. J Adv Nurs 25: 109-116

3. Benbow SM, Jolley DJ (2002) Burnout and stress amongst old age psychiatrists. Int J Geriat Psychiatry 17:710-714

4. Bilgin S, Gozum S (2009) Reducing burnout in mothers with an intellectually disabled child: an education programme. J Adv Nurs 65:2552-5261

5. Boyas J, Wind LH, Kang SY (2012) Exploring the relationship between employment-based social capital, job stress, burnout, and intent to leave among child protection workers: an age-based path analysis model. Child Youth Serv Rev 34(1):50-62

6. Bressi C, Porcellana M, Gambini O, Madia L, Muffatti R, Peirone A, Carlo Altamura A (2009) Burnout among psychiatrists in Milan: a multicenter survey. Psychiatr Serv

7. Brewer EW, Clippard LF (2002) Burnout and job satisfaction among student support services personnel. Hum Resour Dev Q 13(2):169-186

8. Bruce SM, Conaglen HM, Conaglen JV (2005) Burnout in physicians: a case for peer-support. Intern Med J 35(5):272-278

9. Caruso CC (2014) Negative impacts of shiftwork and long work hours. Rehabil Nurs 39:16-25

10. Deahl M, Turner T (1997) General psychiatry in no-man's land. Br Psychiatry 171(1):6-8

11. Dyrbye LN, Varkey P, Boone SL, Satele DV, Sloan JA, Shanafelt TD (2013) Physician satisfaction and burnout at different career stages. In Mayo Clinic proceedings (Vol. 88, no. 12, pp. 1358-1367). Elsevier.

12. Felton JS (1998) Burnout as a clinical entity-its importance in health care workers. Occup med(Lond); 48:237-250.

13. Grassi L, Magnani K (2000) Psychiatric morbidity and burnout in the medical profession: an Italian study of general practitioners and hospital physicians. Psychother Psychosom 69(6):329-334

14. Gundersen L (2001) Physician burnout. Ann Intern Med 135(2):145-148

15. Happell B, Martin T, Pinikahana J (2003) Burnout and job satisfaction: a comparative study of psychiatric nurses from forensic and a mainstream mental health service. Int J Ment Health Nurs 12:39-47

16. Honkonen T, Ahola K, Pertovaara M, Isometsä E, Kalimo R, Nykyri E, Lönnqvist J (2006) The association between burnout and physical illness in the general population - results from the Finnish health 2000 study. J Psychosom Res 61(1):59-66

17. Jensen MT, Rundmo T (2015) Associations between work family conflict, emotional exhaustion, musculoskeletal pain, and gastrointestinal problems in a sample of business travelers. Scand J Psychol 56:105-113

18. Korkeila JA, Töyry S, Kumpulainen K, Toivola JM, Räsänen K, Kalimo R (2003) Burnout and self-perceived health among Finnish psychiatrists and child psychiatrists: a national survey. Scand J Public Health 31(2):85-91

19. Kotb AA, Mohamed KAE, Kamel MH, Ismail MAR, Abdulmajeed AA (2014) Comparison of burnout pattern between hospital physicians and family physicians working in Suez Canal University hospitals. The pan African medical journal, 18.

20. Kumar S (2007) Burnout in psychiatrists. World Psychiatry 6(3):186-189

21. Kumar S (2011) Burnout and job satisfaction in New Zealand psychiatrists: a national follow-up study (doctoral dissertation, ResearchSpace@ Auckland)

22. Lee RT, Ashforth B (1990) On the meaning of Maslach's three dimensions of burnout. J Appl Psychol 75(6):743

23. Lee RT, Ashforth B (1996) A meta-analytic examination of the correlates of the three dimensions of job burnout. J Appl Psychol 81(2):123-133

24. Lešić ARA, Petrović-Stefanović N, Peruničić I, Milenković P, Lečić-Toševski D, Bumbaširević MZ (2009) Burnout in Belgrade orthopaedic surgeons and general practitioners: a preliminary report. Acta chirurgica iugoslavica 56(2):53-59

25. Liebenberg AR, Coetzee JF Jr, Conradie HH, Coetzee JF (2018) Burnout among rural hospital doctors in the Western cape: comparison with previous south African studies. Afr J Prm Health Care Fam Med 10(1):e1

26. Lindström C, Åman J, Norberg A (2010) Increased prevalence of burnout symptoms in parents of chronically ill children. Acta Paediatr 99:427-432

27. Maslach C, Jackson S (1986) Maslach burnout inventory manual, 2nd edn. Consulting Psychologist Press, Palo Alto

28. Maslach D, Jackson S, Leiter M et al (1986) Maslach burnout inventory manual, general survey, human services survey, educators survey and scoring guides. Mind Garden, Menlo Park, CA

29. Mears A, Kendall T, Katona C et al (2002) Career intentions in psychiatric trainees and consultants. Royal College of Psychiatrists, London
30. Meier D, Back E, Morrison AL, R. S. (2001) The inner life of physicians and care of the seriously ill. J Am Med Assoc 286:3007-3014

31. Melamed S, Shirom A, Toker S et al (2006) Burnout and risk of cardiovascular disease: evidence, possible causal paths, and promising research directions. Psychol Bull 132(3):327

32. Montero-Marín J, García-Campayo J, Fajó-Pascual M, Carrasco JM, Gascón S, Gili M et al (2011) Sociodemographic and occupational risk factors associated with the development of different burnout types: the crosssectional University of Zaragoza study. BMC Psychiatry 11(1):1

33. Montgomery A, Panagopoulou E, Kehoe I et al (2011) Connecting organisational culture and quality of care in the hospital: is job burnout the missing link? J Health Organ Manag 25(1):108-123

34. Ogresta J, Rusac S, Zorec L (2008) Relation between burnout syndrome and job satisfaction among mental health workers. Croatian Med J 49(3):364-374

35. Olson SM, Odo NU, Duran AM et al (2014) Burnout and physical activity in Minnesota internal medicine resident physicians. J Grad Med Educ 6(4):669-674

36. Öncel S, Özer ZC, Efe E (2007) Work-related stress, burnout and job satisfaction in Turkish midwives. Soc Behav Pers 35(3):317-328

37. Osama MS (2012) Burnout syndrome among a sample of residents of Ainshams university hospital. Doctoral dissertation, Ain Shams University

38. Pérez TC, Andreu AC, Alvarez N et al (2011) Professional burnout and work satisfaction in Spanish allergists: analysis of working conditions in the specialty. J Investig Allergol Clin Immunol 21(1):13-21

39. Prosser D, Johnson S, Kuipers E et al (1999) Mental health, "burnout" and job satisfaction in a longitudinal study of mental health staff. Soc Psychiatry Psychiatr Epidemiol 34(6):295-300

40. Prosser D, Johnson S, Kuipers E et al (1996) Mental health, 'burnout' and job satisfaction among hospital and community-based mental health staff. $\mathrm{Br}$ J Psychiatry 169(3):334-337

41. Reid $Y$, Johnson S, Morant $N$ et al (1999) Explanations for stress and satisfaction in mental health professionals: a qualitative study. Soc Psychiatry Psychiatr Epidemiol 34(6):301-308

42. Renzi C, Tabolli S, lanni A et al (2005) Burnout and job satisfaction comparing healthcare staff of a dermatological hospital and a general hospital. J Eur Acad Dermatol Venereol 19(2):153-157

43. Rey JM, Walter G, Giuffrida M (2004) Australian psychiatrists today: proud of their profession but stressed and apprehensive about the future. Aust N Z J Psychiatry 38(3):105-110

44. Rogers AE, Hwang WT, Scott LD et al (2004) The working hours of hospital staff nurses and patient safety. Health Aff 23(4):202-212

45. Ross J, Jones J, Callaghan P et al (2009) A survey of stress, job satisfaction and burnout among haemodialysis staff. J Renal Care 35:127-133

46. Rupert PA, Morgan DJ (2005) Work setting and burnout among professional psychologists. Prof Psychol Res Pract 36(5):544

47. Sayil I, Haran S., Olmez U. et al. (1997): "Ankara Universitesi Hastanelerinde Caliuan Doktor Ve Hemuirelerin Tukenmiulik Duzeyleri", Kriz Dergisi, Cilt 5, Say 1:2 ss.71-77.

48. Thompson C (1998) The mental state we are in: morale and psychiatry. Psychiatrist 22(7):405-409

49. Vilardaga R, Luoma JB, Hayes SC et al (2011) Burnout among the addiction counseling workforce: the differential roles of mindfulness and values-based processes and work-site factors. J Subst Abus Treat 40(4):323-335

50. Volker R, Bernhard B, Anna K et al (2010) Burnout, coping and job satisfaction in service staff treating opioid addicts-from Athens to Zurich. Stress Health 26(2):149-159

51. Weber A, Jaekel-Reinhard A (2000) Burnout syndrome: a disease of modern societies? Occup Med 50(7):512-517

52. West CP, Dyrbye LN, Erwin PJ, Shanafelt TD (2016). Interventions to prevent and reduce physician burnout: a systematic review and meta-analysis. Lancet volume 388, Issue 10057, P2272-2281.

53. Whittington R (2002) Attitudes toward patient aggression amongst mental health nurses in the 'zero tolerance' era: associations with burnout and length of experience. J Clin Nurs 11:819-825

\section{Publisher's Note}

Springer Nature remains neutral with regard to jurisdictional claims in published maps and institutional affiliations. 